\title{
PDP-12 stimulus control and data collection for lateralized discrimination learning
}

\author{
KIRMACH NATANI \\ Clinical Sciences Division and Crew Technology Division \\ U.S. Air Force School of Aerospace Medicine, Brooks Air Force Base, Texas 78235 \\ and \\ EDWARD ENGELKEN and DOUGLAS THREATT \\ Biometrics Division \\ U.S. Air Force School of Aerospace Medicine, Brooks Air Force Base, Texas 78235
}

\begin{abstract}
A BASIC program that requires a minimum $8 \mathrm{~K}$ core, $32 \mathrm{~K}$ disk, KW12-A real-time clock, eight extended operation eight-mode DEC relays with peripheral interface, and user-generated special-function assembly language subroutines for clock and relay control was prepared, using advanced OS/8 BASIC, for conducting laterality studies. It is assumed that the user's system has a dual tape drive, CRT, and Teletype. Modified IEE readouts have been used for stimulus presentation. Laterality effects and results suggesting application of the procedure in clinical neuropsychological testing have been achieved. This paper describes the rationale for development of the task, summarizes administration procedures, and provides flowcharts of the BASIC program and assembly language special functions, as well as a circuit diagram for the peripheral relay interface.
\end{abstract}

One problem with techniques currently in use for the investigation of cerebral lateralization is that the choice of tasks and stimuli available have not favored the investigations of qualitative or quantitative aspects of cognitive activity in a single hemisphere. Laterality research has primarily emphasized the categorization of stimuli at the perceptual level by requiring identification and equivalence responses as performance measures. For example, the subject's task is often limited to indicating if two stimuli are "the same" or "different," and the majority of the reports in the literature appear to be studies of visual cognition based upon relatively simple stimulus matching and identification tasks. More complex paradigms have usually not been attempted because the prevailing conceptualization of visual cognitive processes in the tachistoscopic paradigm has traditionally excluded more abstract

This report was prepared by personnel of the Biometrics and Clinical Sciences Divisions of the USAF School of Aerospace Medicine, AFSC, Brooks AFB, Texas 78235. Dr. Natani was supported by a National Research Council Postdoctoral Resident Research Associateship in Neuropsychology, Bryce O. Hartm an, Ph.D., Scientific Advisor. When U.S. government drawings, specifications, or other data are used for any purpose other than a definitely related government procurement operation, the government thereby incurs no responsibility and no obligation whatsoever. Opinions and recommendations stated are those of the authors and do not reflect official USAF policy or endorsement. The voluntary informed consent of the subjects used in this research was obtained in accordance with AFR 80-33. This report has been reviewed and cleared for open publication and/or public release by the appropriate Office of Information (OI) in accordance with AFR 190-17 and DoDD 5230.9. The authors express appreciation to SRA Mary Winfree of the Biomedical Cybernetics Branch, Clinical Sciences Division, USAFSAM, for assistance in the preparation of the illustrations for this report. nonvisual cognitive operations that occur after the visual synthesis is completed. In fact, cognitive psychologists often do not use tachistoscopic techniques in a reaction time paradigm because they believe that is an inappropriate method for assessing common aspects of everyday cognitive situations (Neisser, 1967). The primary exceptions to this position have been reaction time studies of attention to stimulus attributes and speeded classification tasks (Kahneman, 1973). The majority of the laterality studies have also been confounded by the use of stimuli and/or instructions designed to elicit responses favoring a given hemisphere. To overcome these problems, a task nonspecific with regard to lateralization was selected. Thus the same instructions can be used for presentations to either hemisphere. It is expected that significant differences in performance on the task can be attributed to information processing differences in the hemispheres that are independent of stimulus and instruction variables.

The task selected was the optional reversal shift paradigm of Kendler and Kendler (1975). The procedure was modified for lateralized tachistoscopic presentation with response time restriction. The initial work was performed using BRS/LVE Foringer Series 200 digital programming equipment for stimulus display control and data collection operations during each trial. The results obtained with the use of that equipment have been described in some detail (Natani, 1977), and additional reports are in preparation (Natani \& Parsons, Note 1). The initial study supported the position of 
many cognitive psychologists that reaction time paradigms constrain cognitive operations to a perceptual level. Nevertheless, laterality effects were demonstrated, and other results suggested that the procedure should be explored in more detail. In particular, further study of individuals who experienced difficulty with the task indicated that measures of discrimination learning obtained under these conditions might have possibilities for use in clinical neuropsychological testing. Recent work with the tachistoscopic lateralized optional shift (TALOS) task, involving the programming of a PDP-12 computer for stimulus presentation and data collection, is described in this paper.

\section{METHOD}

\section{Control and Stimulus Requirements}

TALOS task administration requires fixation control, tachistoscopic stimulus presentation, response and reaction time recording, auditory feedback indicating errors in procedure, visual feedback indicating a correct or an error response immediately after each trial, a change in the type of stimulus presented following correct or error responses, a stimulus pattern change following 10 consecutive correct responses for both initial learning and reversal shift phases, and recording of the shift response pattern during the test phase. Auditory feedback was provided to indicate faulty procedures, including moving the eye off the fixation number prior to stimulus presentation (stimuli are presented monocularly), responding before a stimulus was presented, or waiting too long to respond. A white-noise masking stimulus $\left(70 \mathrm{~dB}\right.$ re $\left.\mathrm{microN} / \mathrm{m}^{2}\right)$ was provided continuously during the course of the task. The subject had $1.5 \mathrm{sec}$ to respond after the stimulus slide was presented. For clinical applications, this may be the maximum useful response window. The effect of a $1-\mathrm{sec}$ limit on performance will be explored in future work. To obtain conceptual responses, a 3- to 5-sec window may be necessary. The intertrial interval (ITI) was randomly varied from 1.5 to $5 \mathrm{sec}$. The ITI is currently limited to a fixed interval of $5 \mathrm{sec}$ by teleprinter speed. A shorter ITI with randomized variation is desirable and will be used when a faster printer is available. The fixation reporting technique of McKeever, Suberi, and Van Deventer (1972) was used prior to each trial. During the initial study, eye movements activated a Schmitt trigger-controlled buzzer if the subject looked toward the slide screen prior to stimulus presentation. Control voltage for the Schmitt trigger was obtained from bipolar electrodes applied near the temporal orbit (outer canthus) of each eye. Recently, eye movement monitoring has been accomplished by visual observation of the subject's eye and is not linked to computer control.

Special films were prepared for the International Electronic Engineers (IEE) rear-projection readouts used for both warning/ fixation control and stimulus presentation. The central IEE unit (readout locations are illustrated in Figure 1) contains a film that projects a warning/fixation report number $3 \mathrm{~mm}$ high $\times 2 \mathrm{~mm}$ wide before each trial. These small numbers also provided a useful test of visual acuity. The same film is used to project a large $(2.5 \mathrm{~cm}$ high $\times 1.5 \mathrm{~cm}$ wide $)$ green plus or red minus sign for response feedback. The lateralized stimulus readouts (placed $11 \mathrm{deg}$ off the midline to project to the temporal hemiretina) contain a film for presenting two vertically arranged geometric forms covering an area approximately $2.5 \mathrm{~cm}$ high $\times 1.5 \mathrm{~cm}$ wide and subtending visual angles of $2 \mathrm{deg}$ vertically and $1 \mathrm{deg}$ horizontally at a $50-\mathrm{cm}$ viewing distance. For some problems, the two forms that are projected together must be in different colors. At the time the task was developed, multicolored films for IEE readouts were not

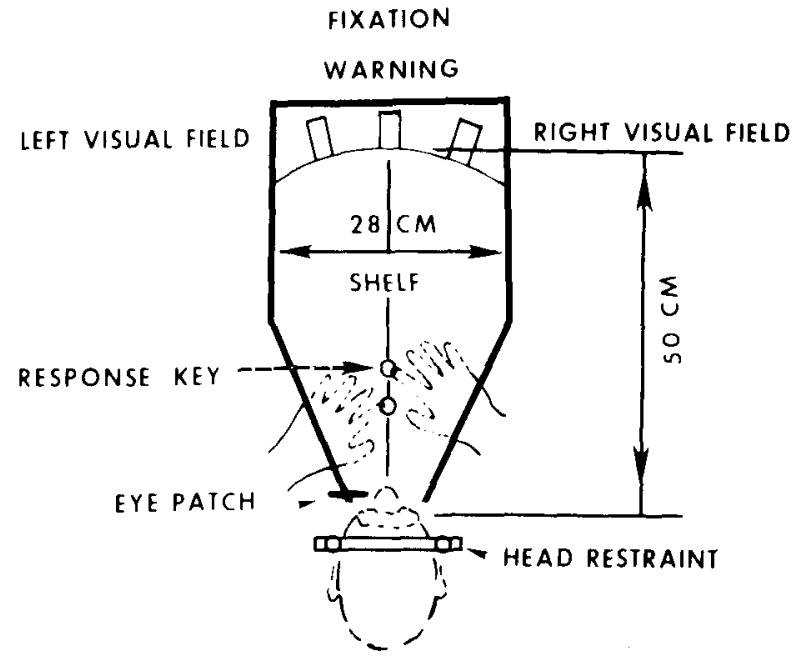

Figure 1. Diagram of the stimulus rear-projection units in relation to the subject and the stimulus presentation and response console. The subject places his chin on a chinrest and his head is firmly clamped in a restraint. Response hands on the keys are not visible to the subject. The projectors in the right and left visual fields are $11 \mathrm{deg}$ from the center warning/fixation stimulus projector.

available commercially. Special films were produced by using a standard black-and-white film, supplied by IEE, to produce a template for the colored stimuli. A standard film containing large numbers was removed from a readout and placed in a glass slide holder for projection in a $70-\mathrm{mm}$ slide projector. The image of the entite number film insert for the readout was projected on a sheet of white poster board, and the area occupied by each number in the film was outlined. The locations of the plane of the board and the projected film were noted. Colored stimuli were then prepared on the poster board within

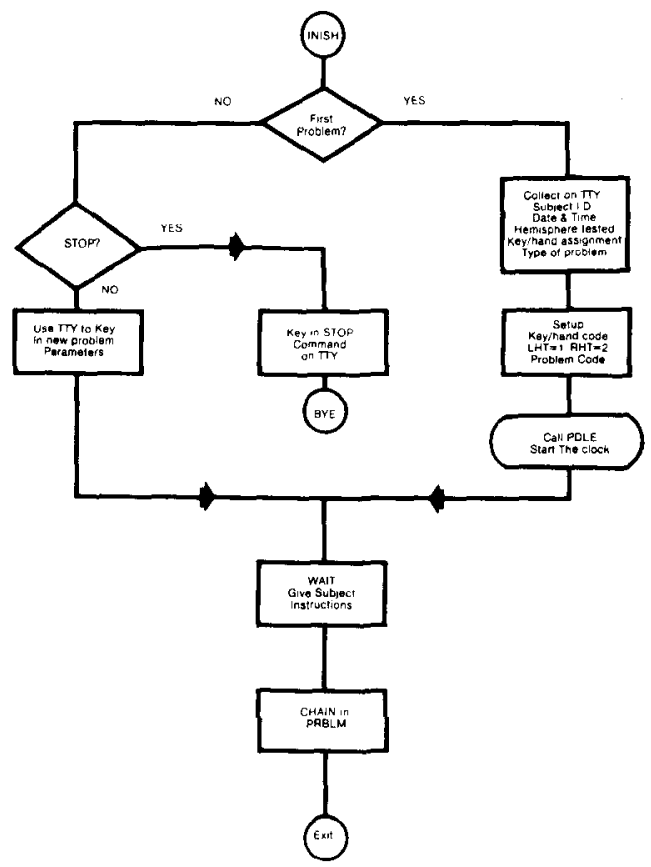

Figure 2. Flowchart of INISH, the OS/8 BASIC start-up and executive routine used to administer the TALOS procedure. 


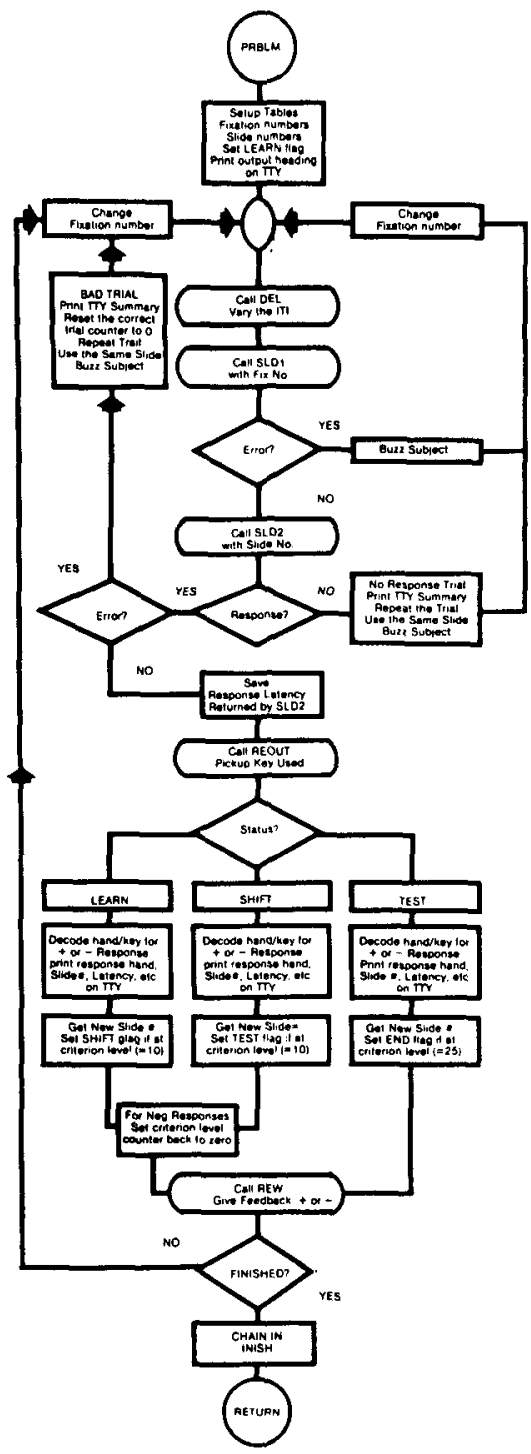

Figure 3. Flowchart of PRBLM, the OS/8 BASIC problem routine used to administer the TALOS procedure. A separate routine such as the one illustrated here is required for each set of stimuli composing a problem.

the areas outlined. The completed stimuli were then photographed in color, using a $35-\mathrm{mm}$ single-lens reflex camera. Bullseye levels and an architectural viewing screen in the camera facilitated the color transparency photography. Care was taken to assure that the plane of the board and the plane of the film in the camera were in exactly the same positions used when the template was drawn. Thus the camera film frame was placed to occupy the same position as the number film during projection for the template preparation. The sprocket holes in the developed $35-\mathrm{mm}$ film were covered on both sides with 3M "magic tape" Type 810 , painted black with India ink, and trimmed to fit the IEE film carrier. The IEE units were also modified for quick film changes by removing the metal next to the optical system on one side. Holes were made above and below the optics to allow the entire optical assembly to be removed for rapid film changes without having to remove the IEE device from the stimulus display console. Further modification of these units for use with film strips and electromechanical film advance are being considered. To provide greater task flexibility, image consistency, and quality, it is highly desirable to generate the stimuli with a microprocessorcontrolled graphics program and display them on a large masked color television set. The possibility of using such a procedure will be explored if future results with TALOS justify the development costs.

\section{TALOS Software}

Digital Equipment Corporation (DEC) BASIC OS/8 software developed for the PDP-8 and PDP-12 series computers was used. This software provides an advanced version of the BASIC programming language that contains added features such as statement packing, chaining, string manipulation, file-oriented input-output, and the capability of including user-generated assembly language functions. All of these features were used in the development of the TALOS program for the PDP-12. Flowcharts for the two main BASIC language routines, INISH and PRBLM, are presented in Figures 2 and 3, respectively.

The OS/8 BASIC facility, which allows experienced assembly language programmers to interface their own routines with the programming language functions, is essential for the inputoutput demands of the task. The user-generated function feature allows assembly language subroutine control of either common system devices or special-purpose peripheral devices. In this application, both capabilities are needed. The KW12-A real-time clock (DEC, 1972) controls slide presentation time and provides subject response latencies in milliseconds. Response key closure provides a $+5-\mathrm{V}$ signal to the KW12-A real-time interface to interrupt the clock for response latency measures. Eight of the extended eight-mode operation, optional expanded relays (DR12-XM), control slide selection and presentation via a special peripheral logic interface with an independent power supply. Figure 4 presents the circuit diagram for the relay

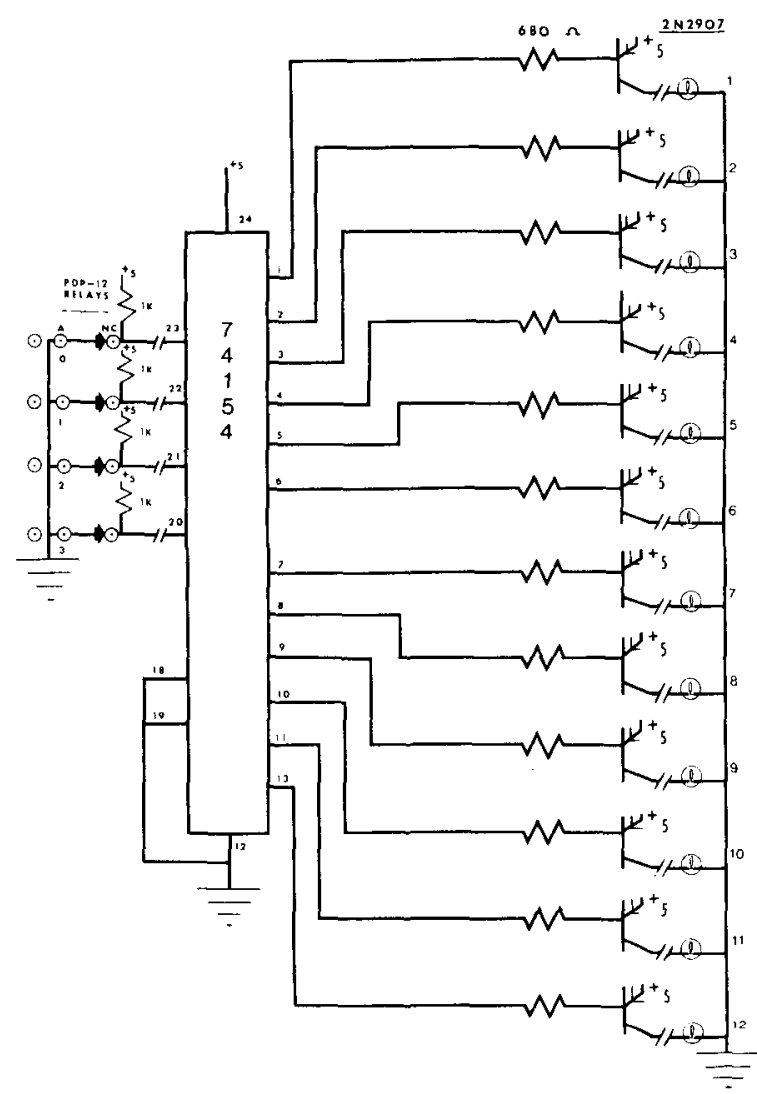

Figure 4. Circuit diagram of logic chip interface used to control stimulus presentation via the PDP-12 extended operation eight-mode expanded relays DR12-XM. The SONALERT buzzer is attached by similar circuitry to Pin 14 of the 74154 chip. 


\begin{tabular}{|c|c|c|c|c|c|c|}
\hline & & 74 & & INPUT & & $\begin{array}{r}74154 \\
\text { OUTPUT }\end{array}$ \\
\hline 3 & 2 & 1 & 0 & OCIAl & $D E C$ & LIGHT \\
\hline 0 & 0 & 0 & 0 & 0 & 0 & 1 \\
\hline 0 & 0 & 0 & 1 & 1 & 1 & 2 \\
\hline 0 & 0 & 1 & 0 & 2 & 2 & 3 \\
\hline 0 & 0 & 1 & 1 & 3 & 3 & 4 \\
\hline 0 & 1 & 0 & 0 & 4 & 4 & 5 \\
\hline 0 & 1 & 0 & 1 & 5 & 5 & 6 \\
\hline 0 & 1 & 1 & 0 & 6 & 6 & 7 \\
\hline 0 & 1 & $\uparrow$ & 1 & 7 & 7 & 8 \\
\hline 1 & 0 & 0 & 0 & 10 & 8 & 9 \\
\hline 1 & 0 & 0 & 1 & 11 & 9 & 10 \\
\hline 1 & 0 & 1 & 0 & 12 & 10 & 11 \\
\hline 1 & 0 & 1 & 1 & 13 & 11 & 12 \\
\hline 1 & 1 & 0 & 0 & 14 & 12 & $B \cup Z$ \\
\hline 1 & 1 & 0 & 1 & 15 & 13 & - \\
\hline 1 & 1 & 1 & 0 & 16 & 14 & - \\
\hline 1 & 1 & 1 & 1 & 17 & 15 & - \\
\hline
\end{tabular}

Figure 5. Truth table indicating relay closure positions used to control logic chip interface to generate up to 16 different control states from four relays.

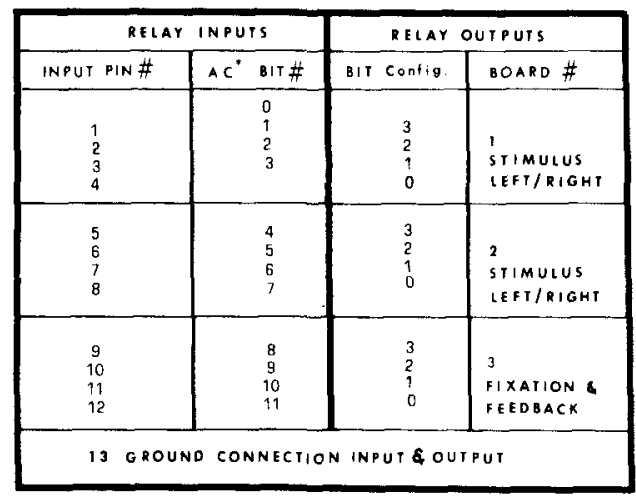

Relays are normally ClOSED $\therefore$

BITO = CLOSED: BIT I = OPEN

IOT 6173 AC TO RELAYS, CLEAR AC

6174 RELAYS TO AC, INCLUSIVE OR

6176 RELAYS TO AC; JAM TRANSFER

Figure 6. Input-output assignments for PDP-12 relays and interface boards.

interface. Figure 5 contains the truth table used to generate up to 16 different control states using four relays to provide input to the 74154 chip (4-line to 16-line decoder). Figure 6 presents the relay input-output assignments with the inputoutput transfer codes (IOTs) used to set accumulator (AC) bit patterns for the DR12-XM relays included, since it has been extremely difficult to obtain documentation for these devices.

User-generated special functions coded into the BASIC UF overlay module of the BASIC run-time system (BRTS) allow up to 12 user functions (DEC, 1974). A UDEF statement in the calling program is used to define the functions within the program. The names given to the functions in the BASIC language program and the assembly language routine need not be the same, since the position of the function in the UDEF statement determines the BRTS location that will be addressed when the special function is used. Currently, 6 of the 12 possible special-function locations available are used for TALOS control: PDLE, DEL, DELY, SLD1, SLD2, REOUT, and REW. PDLE, which is called only once for each job, starts the KW12-A clock and initiates the other functions. Program and ITI pacing is provided by DEL, which delays operation for up to $4 \mathrm{sec}$ each time it is called. Figure 7 contains flowcharts of PDLE and DEL assembly language special functions. DELY provides delay timing during the actual execution of the task and is called by other special functions (see Figure 8). SLD1 (also flowcharted in Figure 8) is called with the warning/fixation
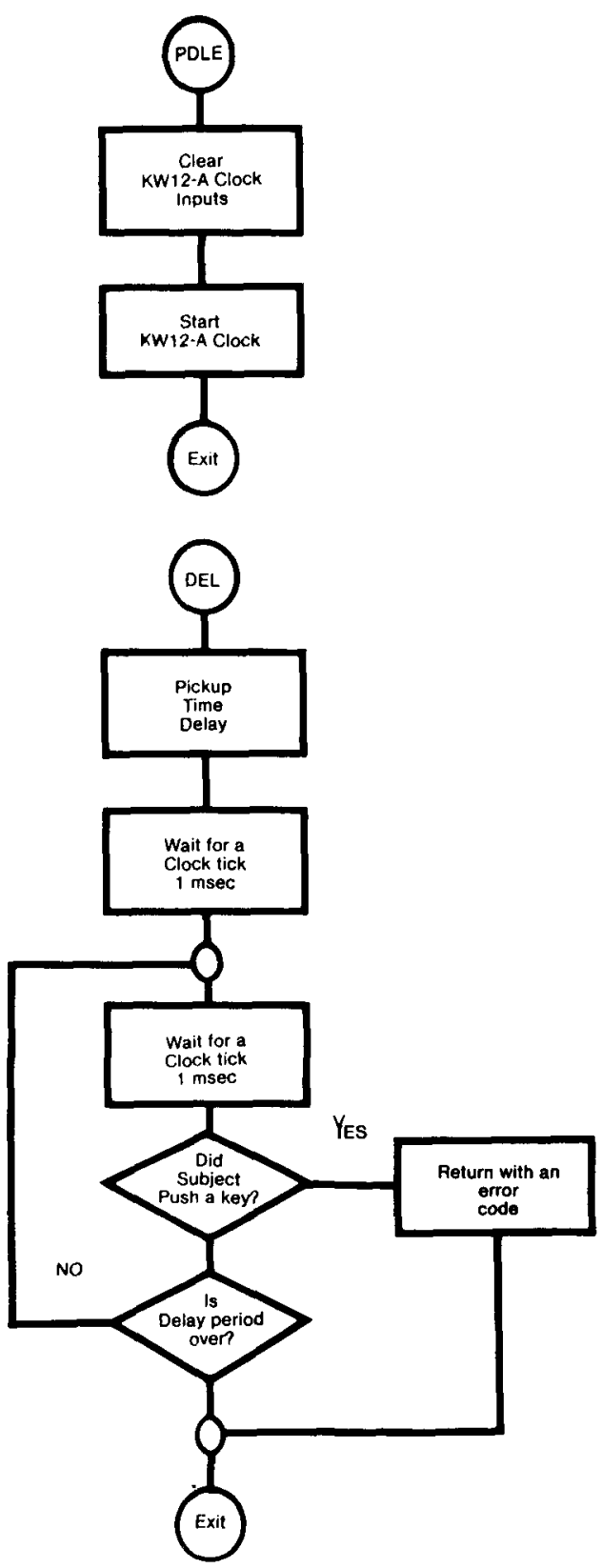

Figure 7. Flowcharts of user-generated special-function assembly language subroutines PDLE and DEL. 


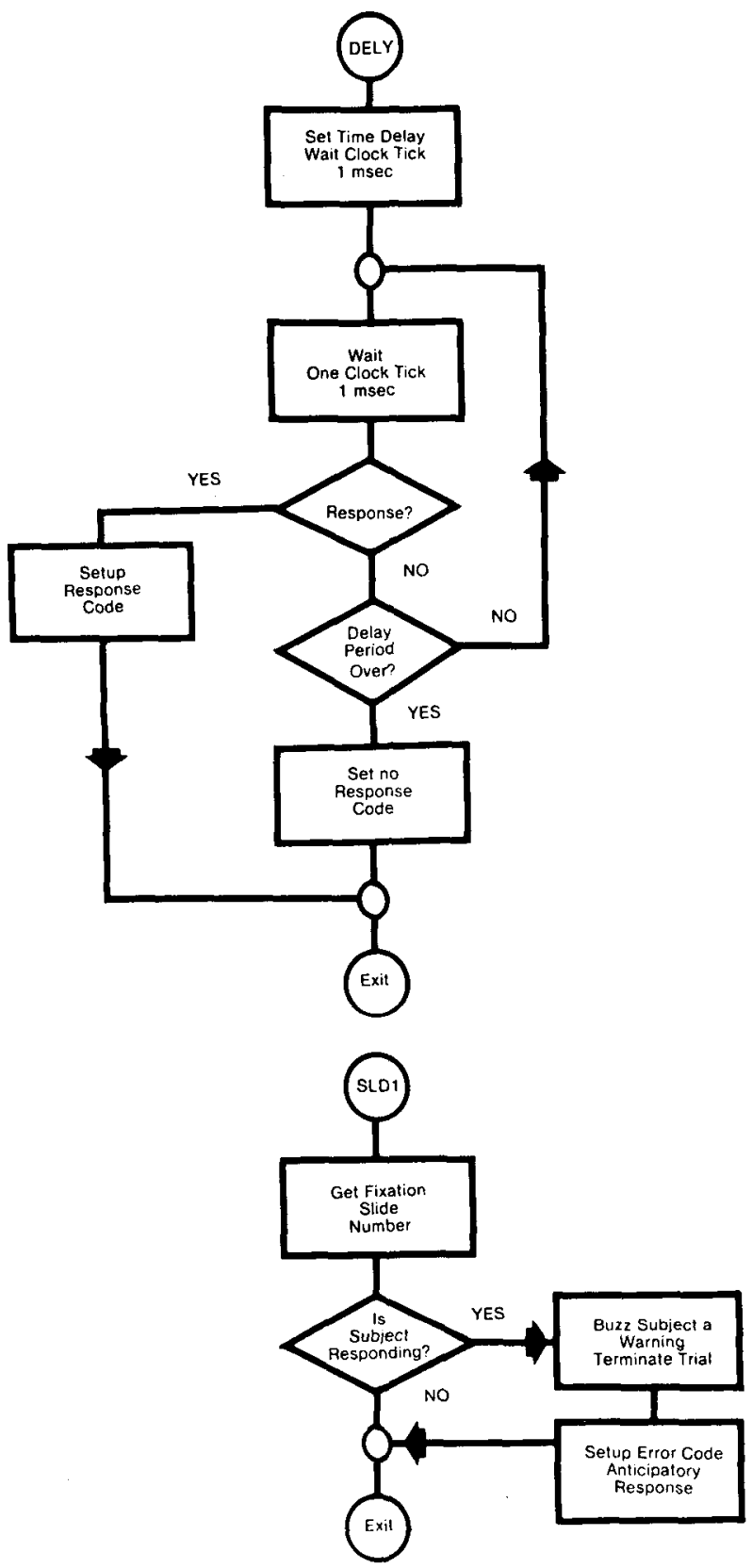

Figure 8. Flowcharts of user-generated special-function assembly language subroutines DELY and SLD1.

slide number to be presented and checks for stuck switches (e.g., subject holding a key down between trials). In the past both hands have been used to obtain responses. Future work will explore the effects of obtaining all responses from a single hand. The routine SLD2 performs most of the work during each trial (see Figure 9). SLD2 is called with the number of the stimulus slide to be presented and controls all presentations during a trial. The routine returns with the subject's response latency. REOUT is used to determine which response key the subject used via the KW12-A clock real-time interface. Calling REW with a slide number provides the subject with positive or negative feedback, depending on the slide designated, after each response. REW is also used to buzz the subject with a SONALERT audible signal if a response is made at an inappropriate time. Flowcharts for REOUT and REW are provided in Figure 10.

\section{TALOS Administration}

Subjects are instructed to begin the initial discrimination learning task without practice by guessing which of the two stimuli presented together is "correct." (See Figure 11 for an illustration of one of the films prepared for the IEE readouts.) A green plus or red minus sign replaces the warning/fixation number within milliseconds after the response key is pressed to provide feedback indicating correct or incorrect responses. Incorrect responses are followed by a change in warning/fixation

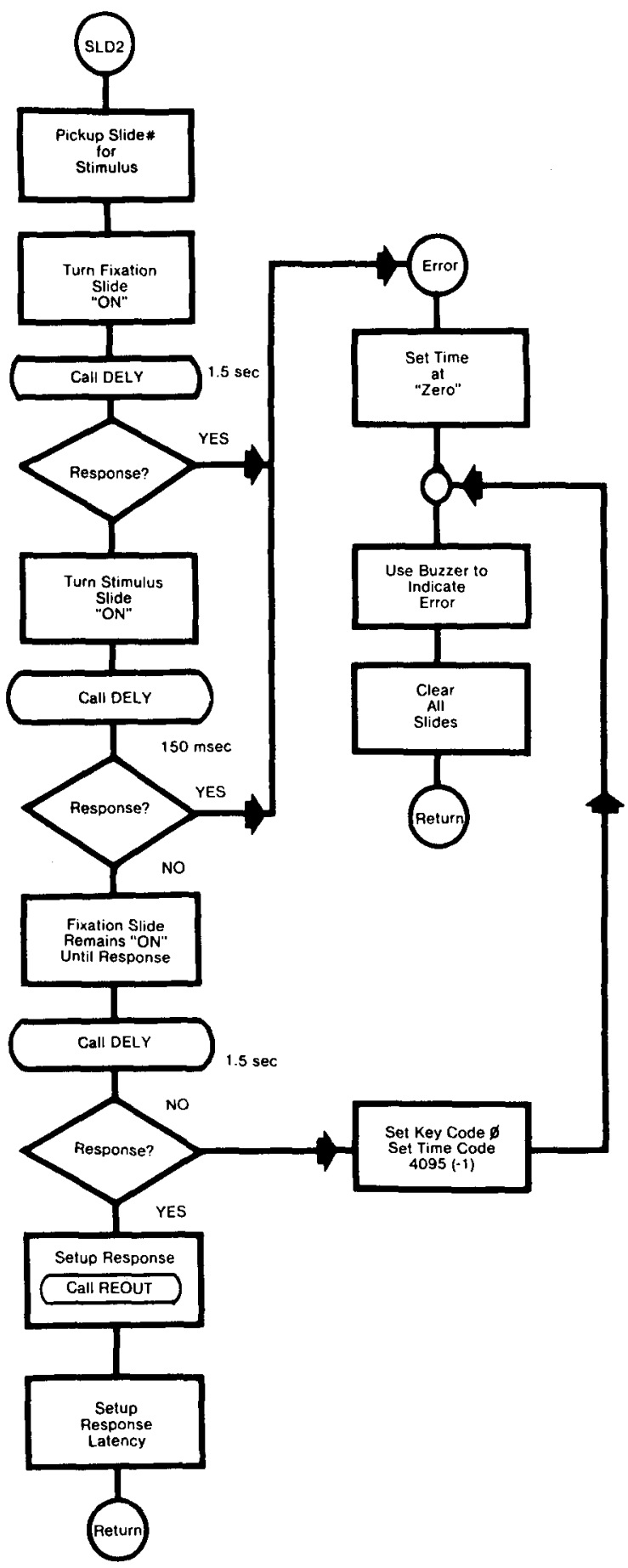

Figure 9. Flowchart for SLD2, the main user-generated special-function assembly language subroutine. This routine controls all presentations during a trial. 


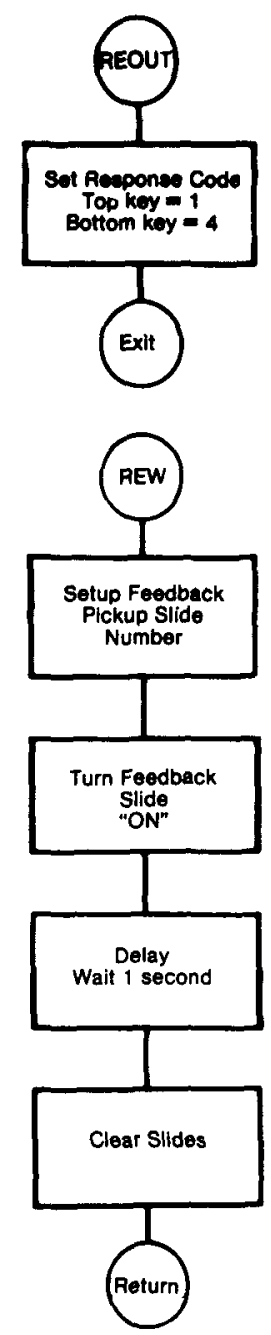

Figure 10. Flowcharts of user-generated special-function assembly language subroutines REOUT and REW.

number on the next trial, but the stimulus slide is not changed and is presented repeatedly until a correct response is obtained. The next slide in the series is then presented, and this procedure is continued until the subject reaches a criterion of 10 successive correct responses. The reversal shift is then introduced without warning. The leaming procedure is repeated until another set of 10 consecutive correct responses is obtained. The testing procedure is then initiated and continued until 25 trials have been completed. Figure 12 illustrates stimulus slides with feedback designation for each of the three phases of the task.

\section{RESULTS}

Because one simple set of instructions can be used for testing either hemisphere with a number of different stimulus sets, the task has potential for the study of both qualitative and quantitative differences in information processing between the two hemispheres of the human brain. Use of the procedure in its original digital logic form revealed significant differences between the left and right hemispheres of human subjects for the initial discrimination learning phase of the task (Natani, 1977). Preliminary use of the PDP-12 version suggests that results comparable to those of the original task can be obtained with much greater facility and reliability. The subject has the option of performing either a concrete perceptual, or an abstract conceptual, solution

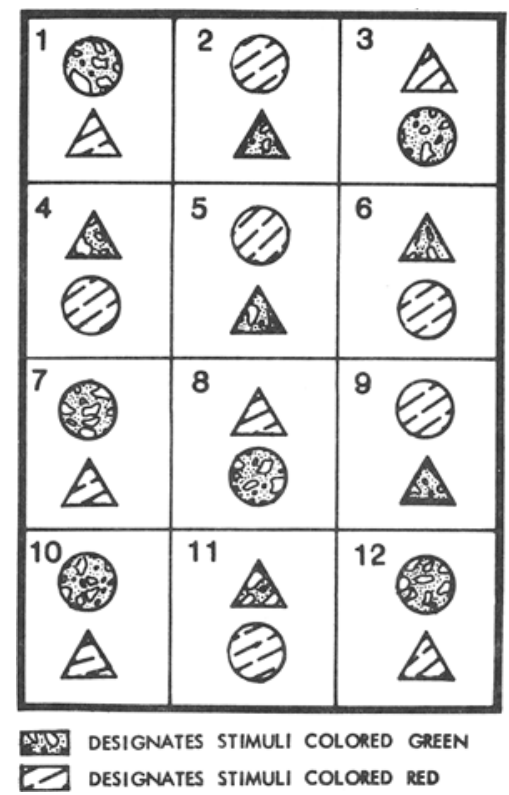

Figure 11. Sample array of bidimensional stimulus compounds used for a color-form discrimination learning task. The relevant concept is shape; position is irrelevant. In this study the subject first learned that circles were correct. Triangles then became correct in the shift learning phase of the task. A reversal shift requires that the subject stay with form during the shift instead of going to color or using some other nonselective strategy.

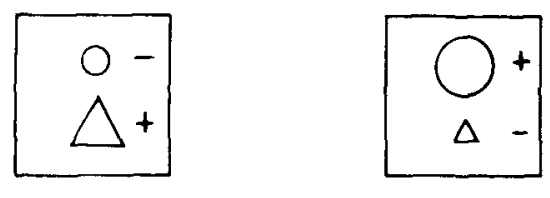

INITIAL DISCRIMINATION LEARNING STIMULUS PAIRS

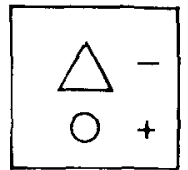

SHIFT LEARNING STIMULI

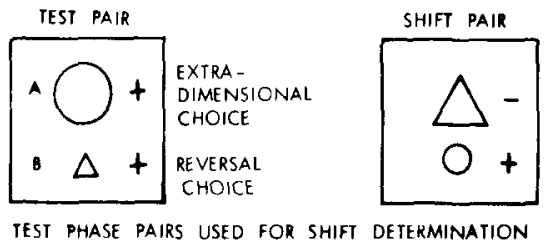

Figure 12. An illustration of the assignment of two bidimensional stimulus pairs during the three phases of the optional shift task. The relevant concept is size. Initial discrimination learning is for large; reversal learning is for small. An extradimensional shift would be indicated by the subject's choice of only circles during the test phase. 
to a simple discrimination learning problem. Most subjects tested appear to have used the perceptual solution. Factors that produce apparent conceptual solutions by some subjects require further exploration. These individual differences, as well as further study of subjects who experienced difficulty with the task, suggested that the procedure has some potential for use in clinical neuropsychological assessment. Preliminary results indicate that the procedure is a reliable research tool and may be useful in exploring drug effects (Natani, Note 2) and in identifying as well as localizing chronic effects of subtle brain damage resulting from closed head injuries (Natani, Note 3). If additional research justifies routine application of the procedure in a clinical setting, microprocessor control with stimuli generated on a masked color television set will be an essential development.

\section{SUMMARY}

The BASIC program TALOS contains an executive routine that interacts with the operator to collect subject ID data, date, time, and type of problem to be run, while separate problem routines control actual task presentation. The executive routine chains an experimental problem control routine from tape. Separate executive and multiple problem routines prevent core overflow, and statement packing in the coding of each routine prevents exceeding the BASIC symbol table capacity. The problem control routines call user-generated machine language special functions to control a real-time clock and operate relays for stimulus presentation and data collection. The relays interface with a 4- to 16-line decoder chip to operate the bulbs in IEE rear-projection readouts. The readouts contain custom stimulus films and have been modified for quick film changes. Preliminary work with the task has demonstrated differences in information processing between the two cerebral hemispheres in human subjects.

\section{REFERENCE NOTES}

1. Natani, K., \& Parsons, O. A. Laterality effects in tachistoscopic discrimination reversal shift learning. Manuscript in preparation, 1978.

2. Natani, K. Quantity of tobacco used and discrimination learning speed in a lateralized tachistoscopic task. Manuscript in preparation, 1978.

3. Natani, K. History of nonspecific encephalopathy and lateral deficits in discrimination reversal shift learning performance. Manuscript in preparation, 1978.

\section{REFERENCES}

Digital Equipment Corporation. PDP-12 System reference manual. Maynard, Mass: Author, 1972.

Digital Equipment Corporation. OS/8 Handbook. Maynard, Mass: Author, 1974.

Kahneman, D. Attention and effort. Englewood Cliffs, N.J: Prentice-Hall, 1973.

Kendler, HiH. H., \& Kendler, T. S. From discrimination learning to cognitive development: A neobehavioristic odyssey. In W. K. Estes (Ed.), Handbook of learning and cognitive processes (Vol. 1). Hillsdale, N.J: Erlbaum, 1975.

McKeever, W. F., Suberi, M., \& Van Deventer, A. D. Fixation control in tachistoscopic studies of laterality effects. Cortex, 1972, 8, 473-479.

NATANI, K. Laterality effects in a tachistoscopic optional shift task in young adults. Dissertation Abstracts International, 1977, 38, 744 .

NeIsser, U. Cognitive psychology. New York: Appleton-CenturyCrofts, 1967. 